\title{
PURELY INSEPARABLE, MODULAR EXTENSIONS OF UNBOUNDED EXPONENT
}

BY

\section{LINDA ALMGREN KIME}

ABSTRACT. Let $K$ be a purely inseparable extension of a field $k$ of characteristic $p \neq 0$. Sweedler has shown in [2, p. 403] that if $K$ over $k$ is of finite exponent, then $K$ is modular over $k$ if and only if $K$ can be written as the tensor product of simple extensions of $k$. This paper grew out of an attempt to find an analogue to this theorem if $K$ is of unbounded exponent over $k$. The definition of a simple extension is extended to include extensions of the form $k\left[x, x^{1 / p}, x^{1 / p^{2}}, \ldots\right]\left[x^{1 / p^{\infty}}\right]$. If $K$ is the tensor product of simple extensions, then $K$ is modular. The converse, however, is not true, as several counterexamples in $\S 4$ illustrate. Even if we restrict $\left[k: k^{p}\right]<\infty$, the converse is still shown to be false.

Given $K$ over $k$ modular, we construct a field $\bigcap_{i=1}^{\infty} k K^{p^{i}} \otimes M(=Q)$ that always imbeds in $K$ where $M$ is the tensor product of simple extensions in the old sense. In general $K \neq Q$. For $K$ to be the tensor product of simple extensions, we need $K=Q$, and $\bigcap_{i=1}^{\infty} k K^{p^{i}}=k\left(\bigcap_{i=1}^{\infty} K^{p^{i}}\right)$. If for some finite $N, k K^{p^{N}}$ $=k K^{p^{N+1}}$, then we have (by Theorem 11) that $K=Q$. This finiteness condition guarantees that $M$ is of finite exponent. Should $\bigcap_{i=1}^{\infty} k K^{p^{i}}=k$, then we would have the condition of Sweedler's original theorem.

The counterexamples in $\S 4$ will hopefully be useful to others interested in unbounded exponent extensions. Of more general interest are two side theorems on modularity. These state that any purely inseparable field extension has a unique minimal modular closure, and that the intersection of modular extensions is again modular.

1. Modular extensions. All fields under consideration will be purely inseparable extensions of a common ground field $k$. They are to be viewed as contained in a common algebraically closed field (e.g. the algebraic closure of $k$ ). If two fields are said to be linearly disjoint, we mean that they are linearly disjoint over their intersection. A field extension $K$ of $k$ is said to be modular if $K^{p^{i}}$ and $k$ are linearly disjoint for all positive i. $K$ is of exponent $n$ over $k$ if $n$ is the minimal positive number such that $K^{p^{n}} \subset k$. If no such $n$ exists, then $K$ is of unbounded or infinite exponent over $k$. Unless otherwise indicated, the intersection and unions through out are taken from $i$ (or $n$ ) $=1$ to $\infty$.

Received by the editors February 14, 1972.

AMS (MOS) subject classifications (1970). Primary 12F15.

Key words and phrases. Purely inseparable, modular, modular closure, infinite or unbounded exponent, tensor product of simple extensions. 
Lemma 1. Let $\left\{M_{a}\right\}$ be a set of fields such that, for any $M_{a_{1}}, M_{a_{2}} \in\left\{M_{a}\right\}$, there exists an $M_{a_{3}} \in\left\{M_{a}\right\}$ such that both $M_{a_{1}}$ and $M_{a_{2}}$ lie in $M_{a_{3}}$. If each $M_{a}$ and $Q$ are linearly disjoint, then $\bigcup_{a} M_{a}$ and $Q$ are linearly disjoint.

Proof. Given any set $\left\{x_{1}, \cdots, x_{n}\right\} \subset \bigcup_{a} M_{a}$, there exists an $a_{0}$ such that $\left\{x_{1}, \cdots, x_{n}\right\} \subset M_{a_{0}} \in\left\{M_{a}\right\}$. If $\left\{x_{1}, \cdots, x_{n}\right\}$ is linearly dependent over 2 , then by the linear disjointness of $M_{a_{0}}$ and $Q,\left\{x_{1}, \cdots, x_{n}\right\}$ is linearly dependent over $M_{a_{0}} \cap Q$. Hence $\left\{x_{1}, \cdots, x_{n}\right\}$ is linear dependent over $\bigcup_{a} M_{a} \cap Q\left(\supset M_{a_{0}} \cap Q\right)$. Q.E.D.

Lemma 2. Let $\left\{M_{a}\right\}$ be as in Lemma 1. If each $M_{a}$ is modular over $k$, then $\bigcup_{a} M_{a}$ is modular over $k$.

Proof. We must show that $\left(\bigcup_{a} M_{a}\right)^{p^{n}}\left(=\bigcup_{a} M_{a}^{p^{n}}\right)$ and $k$ are linearly disjoint for all $n \geq 1$. Given any $\left\{x_{1}, \cdots, x_{s}\right\} \subset \cup_{a} M_{\alpha}^{p^{n}}$ there exists an $\alpha_{0}$ such that $\left\{x_{1}, \cdots, x_{s}\right\} \subset M_{a_{0}}^{p^{n}}$. If $\left\{x_{1}, \cdots, x_{s}\right\}$ is linearly independent over $k \cap\left(\cup_{a} M_{a}^{p^{n}}\right)$, it is linearly independent over $k \cap M_{a_{0}}^{p^{n}}$. The modularity of $M_{a_{0}}$ over $k$ guarantees that $\left\{x_{1}, \cdots, x_{s}\right\}$ is linearly independent over $k$. Q.E.D.

Proposition 3. $K$ is modular over $k \Leftrightarrow k^{1 / p^{n}} \cap K$ is modular over $k$ for all positive $n$.

Proof. Assume $K$ modular over $k$. We want to show that $\left(k^{1 / p^{n}} \cap K\right)^{p^{i}}$ and $k$ are linearly dis joint for all positive $i$.

Case 1. $n>i$. $\left(k^{1 / p^{n}} \cap K\right)^{p^{i}}=k^{1 / p^{n-i}} \cap K^{p^{i}}$. If $\left\{x_{1}, \cdots, x_{n}\right\} \subset k$ is linearly inde pendent over $K^{p^{i}} \cap k=\left(k^{1 / p^{n-i}} \cap K^{p^{i}}\right) \cap k$, by the modularity of $K$ over $k,\left\{x_{1}, \cdots, x_{n}\right\}$ is linearly independent over $K^{p^{i}}$, hence over $k^{1 / p^{n-i}} \cap K^{p^{i}}$.

Case 2. $n \leq i .\left(k^{1 / p^{n}} \cap K\right)^{p^{i}}=k^{p^{i-n}} \cap K^{p^{i}}$. Since $k^{p^{i-n}} \cap K^{p^{i}} \cap k=$ $k^{p^{i-n}} \cap K^{p^{i}},\left(k^{1 / p^{n}} \cap K\right)^{p^{i}}$ and $k$ are trivially linearly dis joint.

Conversely, if $k^{1 / p^{n}} \cap K$ is modular over $k$ for any positive $n$, then $\bigcup\left(k^{1 / p^{n}} \cap K\right)=K$ is modular over $k$ by Lemma 2. Q.E.D.

Sweedler has shown in $[2$, p. 408$]$ that any purely inseparable extension $K$ over $k$ of finite exponent is contained in a unique minimal field extension $L$, where $L$ is modular over $k . L$ is called the modular closure of $K$ over $k$. We now extend this definition to the infinite exponent case.

Theorem 4. Let $K$ be a purely inseparable field extension of $k$.

(1) There exists a unique minimal field extension $L \supset K$ where $L$ is modular over $k$.

(2) $L$ is purely inseparable over $k$.

(3) If $K$ is of exponent $n$, then $L$ has exponent $n$ over $k$. 
Proof. Since $k^{1 / p^{i}} \cap K$ has exponent $p^{i}$ over $k$, by $[2$, p. 408$] k^{1 / p^{i}} \cap K$ has a corresponding modular closure $L_{i}$ satisfying (1)-(3). The minimality of each $L_{i}$ guarantees that $L_{1} \subset L_{2} \subset L_{3} \subset \ldots$. By Lemma $2, \bigcup_{L_{i}}=L$ is modular over $k$. $L$ clearly contains $K$, since $k^{1 / p^{i}} \cap K \subset L_{i}$ and $K=\bigcup^{i}\left(k^{1 / p^{i}} \cap K\right)$. Any field extension of $K$ that is modular over $k$ must contain all the $I_{i_{i}}$ (by the minimality of each $L_{i}$ ). Thus $L$ must be the unique minimal such field. Q.E.D.

Naturally $L$ is called the modular closure of $K$ cver $k$.

Corollary 5. Let $\left\{M_{a}\right\}$ he a set of fields such that each $M_{a}$ is modular over a common ground field $k$. Then $\bigcap_{a} M_{a}$ is modular over $k$.

Proof. Let $N$ be the modular closure of $\bigcap_{a \in A} M_{\alpha}$. By minimality, $N \subset M_{a}$ for all $\alpha \in A$. Hence $N \subset \bigcap_{a} M_{a}$. Q.E.D.

2. A structure theorem for field extensions of unbounded exponent.

Lemma.6. If $K$ over $k$ is modular, then $k\left(k^{1 / p^{i}} \cap K^{p}\right)=k^{1 / p^{i}} \cap k K^{p}$ for any' positive $i$.

Proof. $K=\bigcup\left(k^{1 / p^{i}} \cap K\right) \Rightarrow K^{p}=\bigcup\left(k^{1 / p^{i}} \cap K^{p}\right) \Rightarrow k K^{p}=\bigcup k\left(k^{1 / p^{i}} \cap K^{p}\right)$. If $\left\{x_{n}\right\}$ is a $p$-basis for $k^{1 / p^{n}} \cap K^{p}$ over $k^{1 / p^{n-1}} \cap K^{p},\left\{x_{n}\right\}$ is $p$-independent over $k^{1 / p^{n-1}} \cap k K^{p} \Leftrightarrow\left\{x_{n}^{p^{n-1}}\right\}$ is $p$-independent over $k \cap k^{p^{n-1}} K^{p^{n}}$. $\left\{x_{n}^{p^{n-1}}\right\}\left(\subset\left(k^{1 / p} \cap K^{p^{n}}\right) \subset K^{p^{n}}\right)$ is by assumption $p$-independent over $\left(k^{1 / p^{n \cdots 1}} \cap K^{p}\right)^{p^{n-1}}-k \cap K^{p^{n}}$, so by the linear disjointness of $k$ and $K^{p^{n}}$, $\left\{x_{n}^{p^{n-1}}\right\}$ is $p$-independent over $k$, hence $p$-independent over $k \cap k^{p^{n-1}} K^{p^{n}}$. So $\left\{x_{n}\right\}$ is $p$ independent over $k^{1 / p^{n-1}} \cap k K^{p}$. We also have that $\left\{x_{n}^{p}\right\} \subset\left(k \cap K^{p}\right)\left[\left\{x_{1}\right\},\left\{x_{2}\right\}, \cdots,\left\{x_{n-1}\right\}\right]$ $\subset k\left[\left\{x_{1}\right\}, \cdots,\left\{x_{n-1}\right\}\right]$ where each $\left\{x_{i}\right\}$ is a $p$-basis for $k^{1 / p^{i}} \cap K^{p}$ over $k^{1 / p^{i-1}} \cap K^{p}$.

$$
\left.k K^{p}=: k^{1 / p^{i}} \cap k^{p}\right)=k\left[\left\{x_{1}\right\},\left\{x_{2}\right\}, \ldots\right]
$$

where each $\left\{x_{i}\right\} \in k^{1 / p^{i}} \cap k K^{p}$ is $p$-independent over $k^{1 / p^{i-1}} \cap k K^{p}$,

$$
\begin{aligned}
& \left\{x_{i}^{p}\right\} \subset k\left[\left\{x_{1}\right\},\left\{x_{2}\right\}, \cdots,\left\{x_{i-1}\right\}\right] \\
& \begin{aligned}
& \Rightarrow \text { each }\left\{x_{i}\right\} \text { is a } p \text {-bas is for } k^{1 / p^{i}} \cap k K^{p} \text { over } k^{1 / p^{i-1}} \cap k K^{p} \\
& \Rightarrow k^{1 / p^{i}} \cap k K^{p}=k\left[\left\{x_{1}\right\},\left\{x_{2}\right\}, \cdots,\left\{x_{i}\right\}\right] \\
&=k\left[\left(k \cap K^{p}\right)\left[\left\{x_{1}\right\}, \cdots,\left\{x_{i}\right\}\right]=k\left[k^{1 / p^{i}} \cap K^{p}\right]\right. \text {. Q.E.D. }
\end{aligned}
\end{aligned}
$$

Proposition 7. If $K$ over $k$ is a modular extension then $k K^{p^{n}}$ is modular over $k$ for any positive $n$.

Pronf. Let $\left\{y_{1}\right\},\left\{y_{2}\right\}, \cdots,\left\{y_{i+1}\right\}$ be a modular basis for $k^{1 / p^{i+1}} \cap K$ over $k$, i.e., $k^{1 / p^{i+1}} \cap K=k\left[\left\{y_{1}\right\}\right] \otimes k\left[\left\{y_{2}\right\}\right] \otimes \cdots \otimes k\left[\left\{y_{i+1}\right\}\right]$ where, for each $y_{j} \epsilon$ $\left\{y_{j}\right\}, y_{j}$ has exponent $j$ over $k$. Then 


$$
\begin{aligned}
& k^{1 / p^{i}} \cap k K^{p}=k\left[k^{1 / p^{i}} \cap K^{p}\right](\text { by Lemma 6) } \\
&=k\left[\left(k^{1 / p^{i+1}} \cap K\right)^{p}\right]=k\left[\left\{y_{1}^{p}\right\}, \cdots,\left\{y_{i+1}^{p}\right\}\right] \\
&=k\left[\left\{y_{2}^{p}\right\}\right] \otimes \cdots \otimes k\left[\left\{y_{i+1}^{p}\right\}\right] \\
& \Rightarrow k^{1 / p^{i}} \cap k K^{p} \text { is modular over } k \text { for any } i \geq 0 \\
& \Rightarrow(\text { by Proposition } 3) \text { that } k K^{p} \text { is modular over } k .
\end{aligned}
$$

Now replacing $K$ by $k K^{p}$, we have that $k\left(k K^{p}\right)^{p}=k K^{p^{2}}$ is modular over $k$, hence in general that $k K^{p^{n}}$ is modular over $k$. Q.E.D.

Lemma 8. If $K$ is relatively perfect over $k$, i.e. $K=k K^{p}=k K^{p^{i}}$ for all positive $i$, and $K$ is modular over $k$, then $k^{1 / p} \cap K=k^{1 / p} \cap k K^{p^{n}}=k\left[k^{1 / p} \cap K^{p^{n}}\right]$ for any positive $n$.

Proof. Since $K$ is modular over $k$, we have from Lemma 6 that $k^{1 / p^{i}} \cap k K^{p}=$ $k\left[k^{1 / p^{i}} \cap K^{p}\right]$ for any $i \geq 0$. Using this and the fact that $K=k K^{p^{n}}$ for any $n \geq 0$, we have that

$$
\begin{aligned}
k^{1 / p} \cap K & =k^{1 / p} \cap k K^{p}=k\left[k^{1 / p} \cap K^{p}\right] \\
& =k\left[\left(k^{p}\right)^{1 / p^{2}} \cap k^{p} K^{p^{2}}\right]=k \cdot k^{p}\left[\left(k^{p}\right)^{1 / p^{2}} \cap k^{p^{2}} K^{p^{3}}\right]=k\left[k^{1 / p} \cap K^{p^{2}}\right] .
\end{aligned}
$$

Applying this argument repeatedly we get that $k^{1 / p} \cap K=k^{1 / p} \cap k K^{p^{n}}=$ $k\left[k^{1 / p} \cap K^{p^{n}}\right]$. Q.E.D.

Proposition 9. If $K$ over $k$ is a modular extension then the relatively perfect subfield $\bigcap k K^{p^{i}}$ is modular over $k$. If $\{x\}$ is a p-basis for $k^{1 / p^{n+1}} \cap$ $\left(\bigcap k K^{p^{i}}\right)$ over $k^{1 / p^{n}} \cap\left(\bigcap_{k K^{p^{i}}}\right)$, then $\left\{x^{p^{n}}\right\}$ is a p-basis for $k^{1 / p} \cap\left(\bigcap_{k K^{p^{i}}}\right)$ over $k$.

Proof. Apply Corollary 5 and Proposition 7 to obtain modularity. For simplicity denote $\bigcap_{k K^{p^{i}}}$ by $L$. The modularity of $L$ over $k \Rightarrow\left\{x^{p^{n}}\right\}$ is $p$-independent over $k$.

$$
\begin{aligned}
& k^{1 / p} \cap L=k^{1 / p} \cap k L^{p^{n}}=k\left[k^{1 / p} \cap L^{p^{n}}\right] \quad \text { (by Lemma 6) } \\
& =k\left[k^{1 / p^{n+1}} \cap L\right]^{p^{n}}=k\left[\left(k^{1 / p^{n}} \cap L\right)[\{x\}]\right]^{p^{n}} \\
& =k\left(k \cap L^{p^{n}}\right)\left[\left\{x^{p^{n}}\right\}\right]=k\left[\left\{x^{p^{n}}\right\}\right] \text { Q.E.D. }
\end{aligned}
$$

We note that a relatively perfect field extension $K$ over $k\left(K=k K^{p}=\bigcap k K^{p^{i}}\right)$ is not necessarily modular, as the following example shows.

Let $k=\mathbf{Z}_{3}\left(x^{p}, y^{p}, z^{p^{2}}\right), x, y, z$ indeterminates. Denote any set $\left\{w, w^{1 / p}, w^{1 / p^{2}}, \ldots\right\}$ by $w^{1 / p^{\infty}}$. Set $K=k\left[z^{1 / p^{\infty}},(x z+y)^{1 / p^{\infty}}\right] \cdot k^{1 / p^{2}} \cap K$ over $k$ has diagram

$$
\begin{aligned}
& z, x z+y \\
& \hline z
\end{aligned}
$$

(cf. [2, p. 402]) $\Rightarrow k^{1 / p^{2}} \cap K$ over $k$ is not a modularextension. By Proposition $3, K$ is not modular over $k$. 
Lemma 10. If $A, B$ are field extensions of $k$, such that $A$ is modular over $k$ and $k^{1 / p} \cap A, B$ are linearly disjoint over $k$, then $A, B$ are linearly disjoint over $k$.

Proof. If $\left\{x_{n}\right\}$ is a $p$-basis for $k^{1 / p^{n+1}} \cap A$ over $k^{1 / p^{n}} \cap A$, then $\left\{x_{n}\right\}$ is $p$-independent over $\left(k^{1 / p^{n}} \cap A\right)(B)$. Otherw ise $\left\{x_{n}^{p^{n}}\right\}\left(\subset k^{1 / p} \cap A\right)$, which is $p$ independent over $k$, would be $p$-dependent over $\left(k \cap A^{p^{n}}\right)\left(B^{p^{n}}\right) \subset B$, contradicting the linear disjointness of $k^{1 / p} \cap A$ and $B$. So the basis for $A$ over $k$ consisting of monomials of finite products of the $x_{i}$ is a basis for $A \cdot B$ over $B \Rightarrow A \otimes_{k} B$ $\cong A B \Rightarrow A, B$ are linearly disjoint over $k$. Q.E.D.

Theorem 11. Any modular field extension $K$ over $k$, where for some finite $N, k K^{p^{N}}=k K^{p^{N+1}}$, is isomorpbic to $\bigcap k K^{p^{i}} \otimes M$ where $M$ is a modular subfield of $K$ of finite exponent.

Proof. Let $\left\{x_{1}\right\}$ be a $p$-basis for $k^{1 / p^{2}} \cap K$ over $k^{1 / p} \cap K$. By modularity $\left\{x_{1}^{p}\right\}$ is $p$-independent/k. Let $A_{1}$ be a completion of $\left\{x_{1}^{p}\right\}$ to a $p$-basis for $k^{1 / p} \cap K$ over $k . A_{1}$ is not unique, but the choice of $A_{1}$ is independent of the

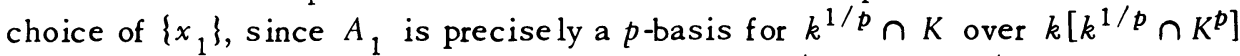
(which equals $k^{1 / p} \cap k K^{p}$ by Lemma 6$)$. Hence $k^{1 / p} \cap K=\left(k^{1 / p} \cap k K^{p}\right) \otimes$ $k\left[A_{1}\right] \Rightarrow k K^{p} \otimes k\left[A_{1}\right] \hookrightarrow K$ (by Lemma 10).

Let $\left\{x_{2}\right\}$ be a $p$-basis for $k^{1 / p^{3}} \cap K$ over $k^{1 / p^{2}} \cap K .\left\{x_{2}^{p}\right\}$ is $p$-independent over $k^{1 / p} \cap K$. Choose $A_{2}$ as any completion of $\left\{x_{2}^{p}\right\}$ to a $p$-basis for $k^{1 / p^{2}} \cap K$ over $k^{1 / p} \cap K$, i.e., a $p$-basis for $k^{1 / p^{2}} \cap K$ over $\left(k^{1 / p} \cap K\right)\left(k^{1 / p^{2}} \cap K^{p}\right)$. $k\left[\left\{x_{2}^{p^{2}}\right\}\right]=k\left[k^{1 / p} \cap K^{p^{2}}\right]=k^{1 / p} \cap k K^{p^{2}}$, so $k^{1 / p} \cap K=\left(k^{1 / p} \cap k K^{p^{2}}\right) \otimes k\left[A_{2}^{p}\right]$ $\otimes k\left[A_{1}\right] \Rightarrow k K^{p^{2}} \otimes k\left[A_{2}\right] \otimes k\left[A_{1}\right] \hookrightarrow K$.

In general, we let $A_{n}$ be a $p$-basis for $k^{1 / p^{n}} \cap K$ over $\left(k^{1 / p^{n-1}} \cap K\right)\left(k^{1 / p^{n}} \cap K^{p}\right) \cdot k^{1 / p} \cap K=\left(k^{1 / p} \cap k K^{p^{n}}\right) \otimes k\left[A_{n}^{p^{n-1}}\right] \otimes \cdots \otimes k\left[A_{1}\right]$ $\Rightarrow k K^{p^{n}} \otimes k\left[A_{n}\right] \otimes \cdots \otimes k\left[A_{1}\right] \hookrightarrow K$. So for any finite $n_{1} k K^{p^{n}}$ and $k\left[A_{1} \cup \ldots \cup A_{n}\right]$ are linearly disjoint over $k \Rightarrow \bigcap k K^{p^{i}}$ and $k\left[A_{1} \cup \cdots \cup A_{n}\right]$ are linearly disjoint over $k$. By Lemma $1, \cup k\left[A_{1} \cup \ldots \cup A_{n}\right]=k\left[A_{1} \cup A_{2} \cup \ldots.\right]$ and $\bigcap k K^{p^{i}}$ are linearly disjoint over $k$, i.e., $\bigcap k K^{p^{i}} \otimes k\left[A_{1} \cup A_{2} \cup \ldots\right]$ imbeds in $K$.

By assumption, there exists a finite $N$ such $k K^{p^{N}}=k K^{p^{N+1}}$, so $A_{i}=\varnothing$ for all $i>N$. Set $M=k\left[A_{1} \cup \cdots \cup A_{N}\right] . M$ is modular since $k\left[A_{1} \cup \cdots \cup A_{N}\right]=$ $k\left[A_{1}\right] \otimes k\left[A_{2}\right] \otimes \cdots \otimes k\left[A_{N}\right](\mathrm{cf} . \S 3)$. We assert that $K=\bigcap k K^{p^{i}} \otimes M$. Let $\left\{x_{1}, \cdots, x_{s}\right\}$ be a $p$-basis for $k^{1 / p^{i+1}} \cap K$ over $k^{1 / p^{i}} \cap K$, where $i>N$. Then $k^{1 / p} \cap K=k\left[x_{1}^{p^{i}}, \cdots, x_{s}^{p^{i}}\right] \otimes k\left[A_{N}^{p^{N}-1}\right] \otimes \cdots \otimes k\left[A_{1}\right]$, where $x_{1}^{p^{i}}, \cdots, x_{s}^{p^{i}}$ are $p$-independent over $k . k\left[x_{1}^{p^{i}}, \cdots, x_{s}^{p^{i}}\right]=k\left[k^{1 / p} \cap K^{p^{i}}\right]=k^{1 / p} \cap k K^{p^{i}}=k^{1 / p} \cap$ $\left(\bigcap k K^{p^{i}}\right)$ (since $\left.i>N\right)$. Proposition 9 says that we may assume that $\left\{x_{1}, \cdots, x_{s}\right\}$ $C k^{1 / p^{i+1}} \cap\left(\bigcap_{k k^{p^{i}}}\right)$. Q.E.D. 
Remark 12. Either condition that $\left[k^{1 / p} \cap K: k\right]<\infty$ or $\left[k: k^{p}\right]<\infty$ implies that, for some $N, k K^{p^{N}}=k K^{p^{N+1}}$. Since if $\left[k^{1 / p} \cap K: k\right]<\infty$ (note $\left[k: k^{p}\right]<\infty$ implies that $\left.\left[k^{1 / p} \cap K: k\right]<\infty\right)$, then $A_{N}=\varnothing$ for some $N \Rightarrow k K^{p^{N}}=k K^{p^{N+1}}$.

With only the restriction that $K$ over $k$ is modular we always have that

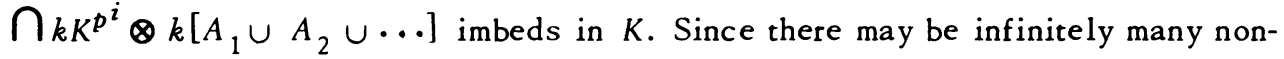
empty $A_{i}$ 's, $k\left[A_{1} \cup A_{2} \cup \ldots\right]$ is not, in general, of finite exponent. We can describe $k\left[A_{1} \cup A_{2} \cup \ldots\right]$ as being of locally finite exponent.

Definition. $K / k$, a purely inseparable extension, is said to be of locally finite exponent if, for any field $L, k \subset L \subset K$, where $\left[k^{1 / p} \cap L: k\right]<\infty$, then $L$ has finite exponent over $k$.

$k\left[A_{1} \cup A_{2} \cup \ldots\right]$ is easily seen to be of locally finite exponent. Given any intermediate field $L$, such that $\left[k^{1 / p} \cap L: k\right]<\infty$, there must exist a finite $n$ such that $L \subset k\left[A_{1} \cup A_{2} \cup \cdots \cup A_{n} \cup A_{n+1}^{p} \cup A_{n+2}^{p^{2}} \cdots\right] \Rightarrow L$ is of exponent $n$ over $k$.

Though we have $\cap k K^{p^{i}} \otimes k\left[A_{1} \cup A_{2} \cup \ldots\right] \hookrightarrow K$, counterexample (d) in $\$ 4$ shows that we need not have $\bigcap k K^{p^{i}} \otimes k\left[A_{1} \cup A_{2} \cup \ldots\right]=K$. We do have the following:

Proposition 13. Given $K$ over $k$ modular, set $Q=\bigcap k K^{p^{i}} \otimes k\left[A_{1} \cup A_{2} \cup \cdots\right]$. Then $K$ is modular and relatively perfect over $Q$ (i.e., $K=\bigcap Q K^{p^{i}}$ ).

Proof. Let $\left\{x_{n}\right\}$ be a $p$-basis for $k^{1 / p^{n}} \cap Q$ over $k^{1 / p^{n-1}} \cap Q .\left\{x_{n}\right\}$ is $p$ independent over $k^{1 / p^{n-1}} \cap K$ (since $Q$ is a modular subfield of $K$ ). If $\left\{y_{n}\right\}$ is a completion of $\left\{x_{n}\right\}$ to a $p$-basis for $k^{1 / p^{n}} \cap K$ over $k^{1 / p^{n-1}} \cap K$, then $\left\{y_{n}^{p}\right\}$ is a possible choice for $\left\{y_{n-1}\right\}$ for all positive $n$ and hence $\left\{y_{1}\right\} \cup\left\{y_{2}\right\} \cup\left\{y_{3}\right\} \cup \cdots$ is a modular basis for $K$ over $Q$. Q.E.D.

Counterexample (e) in $\$ 4$ shows that Theorem 11 cannot be refined by "tensoring off" $k\left[\bigcap K^{p^{i}}\right]$ from $\bigcap k K^{p^{i}}$.

3. A generalization of simple extensions and a tensor product theorem. Sweedler has shown in $[2$, p. 403] that any purely inseparable extension $K$ over $k$ of finite exponent is modular if and only if it is isomorphic to the tensor product (over $k$ ) of simple extensions of $k$. In this section we will study the problem as to whether this theorem has an analogue in the infinite exponent case. We must first extend our definition of a simple extension to include those of the form $k\left[x, x^{1 / p}, x^{1 / p^{2}}, \ldots\right] \equiv k\left[x^{1 / p^{\infty}}\right]$. We may now ask whether a modular extension of infinite exponent is modular if and only if it is isomorphic to the tensor product of simple extensions of $k$.

The following theorem gives us the implication in one direction. 
Theorem 14. If $K$ is isomorphic to the tensor product of simple extensions (of $k$ ), then $K$ is modular over $k$.

Proof. By assumption $K \cong\left(\bigotimes_{\alpha} k\left[x_{\alpha}^{1 / p^{\infty}}\right]\right) \otimes\left(\bigotimes_{\beta} k\left[y_{\beta}\right]\right)$. To prove that $K$ is modular we need to show that $K^{p^{n}}$ and $k$ are linearly disjoint for all positive $n$. Given any $\left\{z_{1}, \cdots, z_{r}\right\} \subset K,\left\{z_{1}, \cdots, z_{r}\right\} \subset\left[\left(\bigotimes_{i=1}^{s} k\left[x_{i}^{1 / p^{u}}\right]\right) \otimes\left(\bigotimes_{j=1}^{t} k\left[y_{j}\right]\right)\right](=Q)$ for some finite $s, t$ and $u, Q$ is modular since it is the tensor product of simple (in the old sense) extensions. Hence if $\left\{z_{1}^{p^{n}}, \ldots, z_{r}^{p^{n}}\right\} \subset K^{p^{n}}$ is linearly dependent over $k$, then, by the linear disjointness of $Q^{p^{n}}$ and $k,\left\{z_{1}^{p^{n}}, \ldots, z_{r}^{p^{n}}\right\}$ is linearly dependent over $Q^{p^{n}} \cap k$, and hence over $K^{p^{n}} \cap k\left(\supset Q^{p^{n}} \cap k\right)$. Thus $K^{p^{n}}$ and $k$ are linearly disjoint. Q.E.D.

It is not true, however, that if $K$ is modular, that it is the tensor product of simple extensions, as counterexamples (a) and (e) of $\$ 4$ demonstrate. Counterexamples (b) and (c) show that even with the added restriction that $\left[k: k^{p}\right]<\infty$, $K$ may still not be the tensor product of simple extensions.

If we restrict $K$ over $k$ such that $k K^{p^{N}}=k K^{p^{N+1}}$ for some $N$ (or $\left[k^{1 / p} \cap K: k\right]<\infty$ or $\left[k: k^{p}\right]<\infty$ ) by Theorem 11 we know that $K \cong \bigcap_{k K^{p^{i}}}$ $\otimes M$, where $M$ is modular (and of finite exponent). Hence $M$ is the tensor product of simple extensions. Since $\bigcap k K^{p^{i}}$ is modular, the following structure theorem shows that if $\bigcap k K^{p^{i}}=k\left[\cap K^{p^{i}}\right]$ then $\bigcap_{k K^{p^{i}}}$ is the tensor product of simple extensions. Thus $K$ will be the tensor product of simple extensions.

Theorem 15. Given a field extension $K$ over $k$, if $k\left[\cap K^{p^{i}}\right]$ is modular, then $k\left[\cap K^{p^{i}}\right] \cong \bigotimes_{a} k\left[z_{a}^{1 / p^{\infty}}\right]$ for some $\left\{z_{a}\right\} \subset \bigcap K^{p^{i}}$.

Proof. Let $\left\{z_{\alpha}\right\} \subset \bigcap K^{p^{i}}$ be a $p$-basis for $k^{1 / p} \cap k\left[\bigcap K^{p^{i}}\right]$ over $k$. Then $\left\{z_{\alpha}^{1 / p^{n}}\right\}$ must be $p$-independent over $k^{1 / p^{n}} \cap k\left[\cap K^{p^{i}}\right]$. If $\left\{z_{\alpha}^{1 / p^{n}}\right\}$ were not a $p$-basis for $k^{1 / p^{n+1}} \cap k\left[\cap K^{p^{i}}\right]$ over $k^{1 / p^{n}} \cap k\left[\cap K^{p^{i}}\right]$, then the modularity of $k\left[\cap K^{p^{i}}\right]$ would imply that $\left\{z_{\alpha}\right\}$ was not a $p$-basis for $k^{1 / p} \cap k\left[\cap K^{p^{i}}\right]$ over $k$. Hence $k^{1 / p^{n}} \cap k\left[\cap K^{p^{i}}\right] \cong \bigotimes_{a} k\left[z_{\alpha}^{1 / p^{n-1}}\right] \Rightarrow k\left[\cap K^{p^{i}}\right] \cong \bigotimes_{\alpha} k\left[z_{\alpha}^{1 / p^{\infty}}\right]$. Q.E.D.

Theorem 16. Given $K$ over $k$ a modular extension, $K$ is the tensor product of simple extensions of $k$ if and only if (in the terminology of Theorem 11) $K \cong$

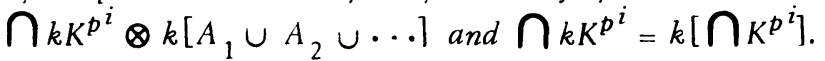

Proof. " $\Longleftarrow "$. The remarks preceding The orem 15 showed that if $K \cong$ $k\left[\cap K^{p^{i}}\right] \otimes k\left[A_{1} \cup A_{2} \cup \ldots\right]$, then $K$ is the tensor product of simple extensions.

" $\Rightarrow "$. Assume that $K \cong\left(\bigotimes_{\alpha} k\left[x_{\alpha}^{\left.1 / p^{\infty}\right]}\right) \otimes\left(\bigotimes_{\beta} k\left[y_{\beta}\right]\right)\right.$. Since each $y_{\beta}$ has

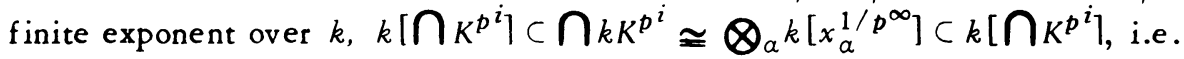
$\bigcap_{k K^{p^{i}}}=k\left[\cap K^{p^{i}}\right]$. 
By Proposition 13 we have that

$$
\begin{aligned}
K & =\bigcap_{n}\left(\bigcap_{i} k K^{p^{i}} \otimes k\left[A_{1} \cup A_{2} \cup \ldots\right]\right)\left(\left(\bigotimes_{a} k\left[x_{a}^{1 / p^{\infty}}\right]\right) \otimes\left(\bigotimes_{\beta} k\left[y_{\beta}\right]\right)\right)^{p^{n}} \\
& =\left(\bigcap_{i} k K^{p^{i}} \otimes k\left[A_{1} \cup A_{2} \cup \ldots\right]\right)\left(\bigotimes_{a}\left[x_{a}^{\left.1 / p^{\infty}\right]}\right)\right. \\
& =\bigcap_{i} k K^{p^{i}} \otimes k\left[A_{1} \cup A_{2} \cup \ldots\right]
\end{aligned}
$$

(since $\left.\bigotimes_{a} k\left[x_{a}^{1 / p^{\infty}}\right] \subset k\left[\bigcap_{i} K^{p^{i}}\right] \subset \bigcap_{i} k K^{p^{i}}\right)$. Q.E.D.

We have shown (Remark 12) that if $k K^{p^{N}}=k K^{p^{N \because 1}}$ for some finite $N$, or $\left[k^{1 / p} \cap K: k\right]<\infty$, or $\left[k: k^{p}\right]<\infty$, then we will have $\left.K \cong \bigcap_{k K^{p^{i}} \otimes k} \otimes A_{1} \cup A_{2} \cup \ldots\right]$. The following theorem gives some conditions under which $\cap k K^{p^{i}}=k\left[\cap K^{p^{i}}\right]$.

Theorem 17. Let $K$ over $k$ be a modular extension.

(1) If $K$ over $\bigcap K^{p^{i}}$ is finitely generated as a field, then $\bigcap k K^{p^{i}}=k\left[\bigcap_{p^{p}}\right]$.

(2) If there exists a generating set for $K$ over $K^{p}$ that generates $K$ over

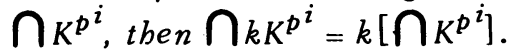

Proof. (1) Assume $K=\left(\bigcap_{i} K^{p^{i}}\right)\left(x_{1}, \ldots, x_{m}\right)$. There exists a finite $n$ such that $x_{i}^{p^{n}} \in k, \quad i=1, \cdots, m$. Hence $K^{p^{j}}=\left(\bigcap_{i} K^{p^{i}}\right)\left(x_{1}^{p^{j}}, \cdots, x_{m}^{p^{j}}\right) \subset k\left(\bigcap_{i} K^{p^{i}}\right)$ for any $j \geq n \Rightarrow k K^{p^{j}}=k\left(\bigcap_{i} K^{p^{i}}\right)$ for any $j \geq n$. Therefore $\bigcap_{i} k K^{p^{i}}=k\left(\bigcap_{i} K^{p^{i}}\right)$.

(2) Assume that $\{x\}$ is a generating set for $K$ over $K^{p}$ such that $K=$ $\left(\bigcap_{i} K^{p^{i}}\right)(\{x\})$. For any positive $n, k K^{p^{n}}=k\left[\left(\bigcap_{i} K^{p^{i}}\right)\left[\left\{x^{p^{n}}\right\}\right]\right]$. Hence if $a \in$ $\bigcap_{n} k K^{p^{n}}, \alpha \in \bigcap_{n}\left(k\left[\left(\bigcap_{i} K^{p^{i}}\right)\left[\left\{x^{p^{n}}\right\}\right]\right]\right)=k\left[\bigcap_{i} K^{p^{i}}\right]$. Q.E.D.

4. Some counterexamples. (a) The following counterexample illustrates that a modular field extension of unbounded exponent need not be a tensor product of simple extensions. In particular we will construct a modular relatively perfect field extension $K$ over $k$ (i.e. $K=\bigcap k K^{p^{i}}$ ), where $K \neq k\left[\bigcap K^{p^{i}}\right]$.

Let $\mathrm{Z}_{p}$ be the prime field of characteristic $p$, and $x_{1}, x_{2}, x_{3}, \cdots$ be indeterminates.

Let

$$
\begin{aligned}
k & =\mathbf{Z}_{p}\left(x_{1}, x_{2}, x_{3}, \cdots\right) \\
k^{1 / p} \cap K & =k\left[x_{1}^{1 / p}\right] \\
k^{1 / p^{2}} \cap K & =k\left[x_{1}^{1 / p^{2}} x_{2}^{1 / p}\right] \\
k^{1 / p^{n}} \cap K & =k\left[x_{1}^{1 / p^{n}} x_{2}^{1 / p^{n-1}} \cdots x_{n}^{1 / p}\right]
\end{aligned}
$$


It is easy to verify that $K$ is of the form $\bigcap k K^{p^{i}}$. We claim that $K \neq k\left[\cap K^{p^{i}}\right]$, in particular that $\cap K^{p^{i}} \subset k$.

Assume not. Then there exists a $y \in \bigcap K^{p^{i}}$ where $y \in k-k^{p}$ (set difference). Then $y$ will be in all of the following fields:

$$
\begin{aligned}
k \cap K^{p} & =Z_{p}\left(x_{1}, x_{2}^{p}, x_{3}^{p}, \ldots\right) \\
k \cap K^{p^{2}} & =Z_{p}\left(x_{1} x_{2}^{p}, x_{1}^{p}, x_{2}^{p^{2}}, x_{3}^{p^{2}}, \ldots\right) \\
& \cdot \\
& \cdot \\
k \cap K^{p^{i}} & =\mathbf{Z}_{p}\left(x_{1} x_{2}^{p} \cdots x_{i}^{p^{i-1}}, x_{1}^{p^{i-1}}, x_{2}^{p^{i}}, x_{3}^{p^{i}}, \ldots\right)
\end{aligned}
$$

$\bigcap\left(k \cap K^{p^{2}}\right) \subset \mathbf{Z}_{p}\left(x_{1}, x_{2}^{p}, x_{3}^{p^{2}}, \ldots\right)$, so $y \in \mathbf{Z}_{p}\left(x_{1}, x_{2}^{p}, \ldots, x_{n}^{p^{n-1}}\right)(=F)$ for some finite $n . y \in k \cap K^{p^{n+1}} \Rightarrow y \in \mathbf{Z}_{p}\left(x_{1} x_{2}^{p} \ldots x_{n}^{p^{n-1}} x_{n+1}^{p^{n}}, x_{1}^{p^{n}}, x_{2}^{p^{n+1}}, \cdots, x_{n+1}^{p^{n+1}}\right)$ $(=G)$.. We claim that $F \cap G=\mathbf{Z}_{p}\left(x_{1}^{p} x_{2}^{p^{2}} \ldots x_{n}^{p^{n}}, x_{1}^{p^{n}}, x_{2}^{p^{n+1}}, \ldots, x_{n}^{p^{n+1}}\right)$. So $y \in F \cap G \subset k^{p}$, which contradicts our hypothes is that $y \in k-k^{p}$.

Consider the following diagram for $\mathrm{Z}_{p}\left(x_{1}, x_{2}^{p}, \cdots, x_{n}^{p^{n-1}}, x_{n+1}^{p^{n}}\right)$ as a field extension of $\mathrm{Z}_{p}\left(x_{1}^{p^{n}}, x_{2}^{p^{n+1}}, \cdots, x_{n}^{p^{n+1}}, x_{n+1}^{p^{n+1}}\right)$.

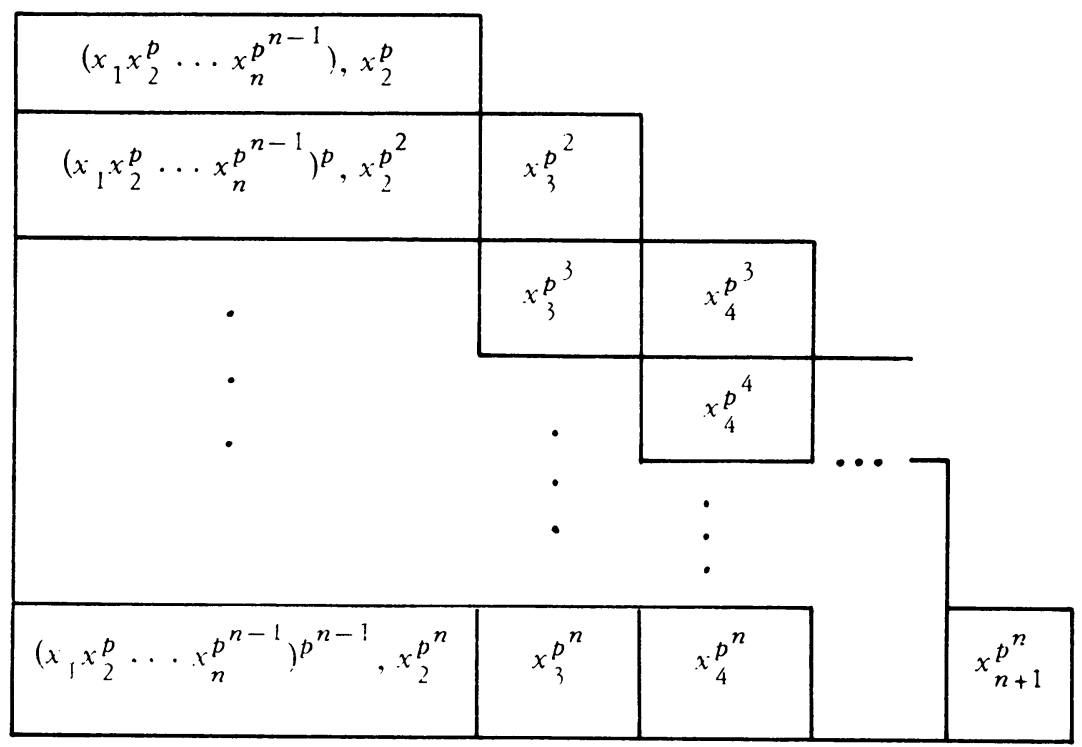

We want to describe $F$ and $G$ using the above diagram. Recall that the ground field for this diagram is $Z_{p}\left(x_{1}^{p^{n}}, x_{2}^{p^{n+1}}, \cdots, x_{n}^{p^{n+1}}, x_{n+1}^{p^{n+1}}\right)$. If we deleted $x_{n+1}^{p^{n}}$ from the diagram and restricted the ground field to $\mathrm{Z}_{p}\left(x_{1}^{p^{n}}, x_{2}^{p^{n+1}}, \cdots, x_{n}^{p^{n+1}}\right)$, the above would be a diagram for $F$. 
The elements of $G$ are those in the span of monomials in $\left(\left(x_{1} x_{2}^{p} \cdots x_{n}^{p^{n-1}}\right) \cdot x_{n+1}^{p^{n}}\right)^{i}$ and $\left(\left(x_{1} x_{2}^{p} \cdots x_{n}^{p^{n-1}}\right)^{p}\right)^{j}$ where $i=0, \cdots, p-1$, $j=0, \cdots, p^{n-1}-1$.

Hence $F \cap G$ can be described as polynomials in $\left(x_{1} x_{2}^{p} \cdots x_{n}^{p^{n-1}}\right)^{p}$ with coefficients in $Z_{p}\left(x_{1}^{p^{n}}, x_{2}^{p^{n+1}}, \ldots, x_{n}^{p^{n+1}}\right)$, i.e.

$$
F \cap G=\mathbf{Z}_{p}\left(x_{1}^{p} x_{2}^{p^{2}} \cdots x_{n}^{p^{n}}, x_{1}^{p^{n}}, x_{2}^{p^{n+1}}, \cdots, x_{n}^{p^{n+1}}\right) \text {. Q.E.D. }
$$

(b) The following argument shows that even with the restriction that $\left[k: k^{p}\right]<\infty$, a modular extension may not always be a tensor product of simple extensions.

Let $k$ be any field of characteristic $p \neq 0$ such that $\left[k: k^{p}\right]>p . k$ by assumption is not perfect, hence in particular $|k|$ is infinite. Assume that $|k|=\boldsymbol{N}_{0}$, and let $L$ be the perfect closure of $k$.

A well-known theorem from Galois theory states that, given any field extension $K$ over $k, K$ is primitive over $k \Leftrightarrow$ there are a finite number of intermediate fields. In the case when $K$ is purely inseparable over $k$ and of exponent one, there are a finite number of intermediate fields $\Leftrightarrow[K: k]=p$.

Lemma 18. Any purely inseparable extension $K$ over $k$ of the form $\cap k K^{p^{i}}$, where $\left[k^{1 / p^{n+1}} \cap K: k^{1 / p^{n}} \cap K\right]=p$, is modular.

Proof. Any such $K$ is of the form $k\left[x_{1}, x_{2}, \ldots\right]$ where $x_{i}$ has exponent $i$ over $k$ and $x_{i}^{p} \in k\left[x_{1}, \cdots, x_{i-1}\right] . k^{1 / p^{n}} \cap K=k\left[x_{1}, x_{2}, \cdots, x_{n}\right]=k\left[x_{n}\right]$ is modular by [2, p. 403]. By Proposition 3, $K$ is modular over $k$. Q.E.D.

Lemma 19. There are at most $|k|=\boldsymbol{\kappa}_{0}$ purely inseparable modular extensions $K$ of the form $k\left[\cap K^{p^{i}}\right]$, where $\left[k^{1 / p} \cap K: k\right]=p$ (i.e., of the form $k\left[z^{1 / p^{\infty}}\right]$ ).

Proof. Trivial, since we may associate any field of the form $k\left[z^{1 / p^{\infty}}\right](z \in k)$ with $z$. Q.E.D.

Theorem 20. There exist $2^{\boldsymbol{K}_{0}}$ purely inseparable modular field extensions of the form $\bigcap_{k K^{p^{i}}}$, where $\left[k^{1 / p} \cap K: k\right]=p$ and $\left[k: k^{p}\right]>p$.

Proof. The field extensions under consideration are of the form $k\left[x_{1}, x_{2}, x_{3}, \ldots\right]$ where $\left[k\left[x_{i}\right]: k\right]=p^{i}$ and $x_{i}^{p} \in k\left[x_{1}, \cdots, x_{i-1}\right]$.

Given any $x_{1} \in\left(k^{1 / p} \cap L\right)-k$, there exists a $y \in\left(k^{1 / p} \cap L\right)-k$ such that $x_{1}$ and $y$ are $p$-independent over $k$ (since $\left[k: k^{p}\right]>p$ ). Consider $M_{1}=k\left[x_{1}^{1 / p}, y\right]$ as a field extension of $k\left[x_{1}\right]$. Since $M_{1}^{p} \subset k\left[x_{1}\right]$ and $\left[M: k\left[x_{1}\right]\right]=p^{2}$, by an earlier remark there are an infinite number of intermediate fields, all of which (excepting $k\left[x_{1}, y\right]$ ) have exponent 2 over $k$. We associate with each such field $N$, an $\alpha$ such that $N=\left(k\left[x_{1}\right]\right)[\alpha]=k[\alpha]$. For any given $\alpha,[k[\alpha]: k]=p^{2}$, and 
if $a \neq \alpha^{\prime}, k\left[x_{1}, \alpha\right]=k[\alpha] \neq k\left[\alpha^{\prime}\right]=k\left[x_{1}, \alpha^{\prime}\right]$. Clearly the $\alpha^{\prime}$ 's provide an infinite number of candidates for an $x_{2}$.

Having chosen an $x_{2}$, we consider $M_{2}=k\left[x_{1}, x_{2}^{1 / p}, y\right]$ as a field extension of $k\left[x_{1}, x_{2}\right] . M_{2}^{p} \subset k\left[x_{1}, x_{2}\right],\left[M_{2}: k\left[x_{1}, x_{2}\right]\right]=p^{2}$, so there exist an infinite number of intermediate fields of degree $p$ over $k\left[x_{1}, x_{2}\right]$ and exponent 3 over $k$.

Each of these provides a candidate for $x_{3}$, such that if $x_{3} \neq x_{3}^{\prime}$, then $k\left[x_{1}, x_{2}, x_{3}\right]$ $\neq k\left[x_{1}, x_{2}, x_{3}^{\prime}\right]$.

We proceed in the above manner to choose an $x_{4}, x_{5}$, etc. For each $x_{i}$ there exist an infinite number of choices such that, if $x_{i} \neq x_{i}^{\prime}, k\left[x_{1}, \ldots, x_{i}\right] \neq k\left[x_{1}, \cdots, x_{i}^{\prime}\right]$. Hence there exist at least $x_{0}^{K_{0}}=2^{X_{0}}$ choices for modular field extensions $K / k$ of the form $\bigcap_{k K^{p}}$, where $\left[k^{1 / p} \cap K: k\right]=p$ and $\left[k: k^{p}\right]>p$. Q.E.D.

(c) In the following example, we will explicitly construct a modular field extension $K$ over $k$, where $K=\bigcap k K^{p^{i}} \neq k\left[\bigcap K^{p^{i}}\right]$ and $\left[k: k^{p}\right]<\infty$.

Let $k=\mathbf{Z}_{p}\left(x, y, z_{1}^{1 / p^{\infty}}, z_{2}^{1 / p^{\infty}}, \ldots\right)$ where $x, y, z_{1}, z_{2}, \cdots$ are indeterminates (note $\left[k: k^{p}\right]=p^{2}$ ).

Let

$$
\begin{aligned}
k^{1 / p} \cap K & =k\left[x^{1 / p}\right] \\
k^{1 / p^{2}} \cap K & =k\left[x^{1 / p^{2}}+z_{1} y^{1 / p}\right] \\
k^{1 / p^{3}} \cap K & =k\left[x^{1 / p^{3}}+z_{1}^{1 / p} y^{1 / p^{2}}+z_{2} y^{1 / p}\right] \\
& \cdot \\
& \cdot \\
k^{1 / p^{n}} \cap K & =k\left[x^{1 / p^{n}}+z_{1}^{1 / p^{n-2}} y^{1 / p^{n-1}}+\cdots+z_{n-1} y^{1 / p}\right]
\end{aligned}
$$

Assume that $K=k\left[\bigcap K^{p^{i}}\right]$. Then there exists an $\alpha \epsilon k-k^{p}$ such that $\alpha \epsilon$ $\bigcap K^{p^{i}}$. a will lie in all of the following fields.

$$
\begin{aligned}
k \cap K^{p} & =Z_{p}\left(x, y^{p}, z_{1}^{1 / p^{\infty}}, z_{2}^{1 / p^{\infty}}, \ldots\right) \\
k \cap K^{p^{2}} & =\mathbf{Z}_{p}\left(x+z_{1}^{p^{2}} y^{p}, y^{p^{2}}, z_{1}^{1 / p^{\infty}}, z_{2}^{1 / p^{\infty}}, \ldots\right) \\
& \cdot \\
& \cdot \\
k \cap K^{p^{i}} & =Z_{p}\left(x+z_{1}^{p^{2}} y^{p}+\cdots+z_{i-1}^{p^{i}} y^{p^{i-1}}, y^{p^{i}}, z_{1}^{1 / p^{\infty}}, z_{2}^{1 / p^{\infty}}, \ldots\right)
\end{aligned}
$$

There exists a finite $n$ such that $\alpha \in \mathbf{Z}_{p}\left(x, y, z_{1}^{1 / p^{\infty}}, \cdots, z_{n}^{1 / p^{\infty}}\right)(=F)$. But $\alpha$ also $\in k \cap K^{p^{n+2}}=\mathbf{Z}_{p}\left(x+z_{1}^{p^{2}} y^{p}+\cdots+z_{n+1}^{p^{n+2}} y^{p^{n+1}}, y^{p^{n+2}}, z_{1}^{1 / p^{\infty}}, z_{2}^{1 / p^{\infty}}, \ldots\right)$ $(=G)$.

Consider the following diagram for $\mathbf{Z}_{p}\left(x, y, z_{1}^{1 / p^{\infty}}, z_{2}^{1 / p^{\infty}}, \ldots\right)$ as a field extension of $\mathrm{Z}_{p}\left(x^{p^{n+1}}, y^{p^{n+2}}, z_{1}^{1 / p^{\infty}}, \cdots, z_{n}^{1 / p^{\infty}}, z_{n+1}^{p^{n+3}}, z_{n+2}^{1 / p^{\infty}}, \cdots\right)$. 


\begin{tabular}{|c|c|c|}
\hline$z_{n+1}^{!} p$ & & \\
\hline$z_{n+1}$ & & \\
\hline$z_{n+1}^{p}$ & $y$ & \\
\hline$z_{n+1}^{p^{2}}$ & $y^{p}$ & $x+z_{1}^{p^{2}} y^{p}+\cdots+z_{n}^{p^{n+i}} y^{p^{n}}$ \\
\hline$\dot{\bullet}$ & $\dot{\bullet}$ & $\dot{\bullet}$ \\
\hline$z_{n+i}^{p^{n+2}}$ & $y^{p^{n+1}}$ & $\left(x+z_{:}^{p^{2}} y^{p}+\cdots+z_{n}^{p^{n+1}} y^{p^{n}}\right)^{p^{n}}$ \\
\hline
\end{tabular}

We would like to use the above diagram to describe $F$ and $G$. If we deleted the left-hand column (i.e. all $p$ th roots of $z_{n+1}^{p^{n+2}}$ ) and restricted the ground field to $\mathbf{Z}_{p}\left(x^{p^{n+1}}, y^{p^{n+2}}, z_{1}^{1 / p^{\infty}}, \cdots, z_{n}^{1 / p^{\infty}}\right)$ the above would be a diagram for $F$.

$G$ consists of the span of monomials in $\left\{\left(\left(z_{n+1}^{p^{n+2}}\right)^{1 / p^{\infty}}\right)^{i}\right\}$, $\left[\left(x+z_{1}^{p^{2}} y^{p}+\cdots+z_{n}^{p^{n+1}} y^{p^{n}}\right)+\left(z_{n+1}^{p^{n+2}} y^{p^{n+1}}\right)\right]^{j}$ and $\left(\left(x+z_{1}^{p^{2}} y^{p}+\cdots+z_{n}^{p^{n+1}} y^{p^{n}}\right)^{p}\right)^{k}$ where $i, j=0, \cdots, p-1, k=0, \cdots, p^{n}-1$. Hence $F \cap G$ can be described as polynomials in $\left(x+z_{1}^{p^{2}} y^{p}+\cdots+z_{n}^{p^{n+1}} y^{p^{n}}\right)^{p}$ with coefficients in $\mathbf{Z}_{p}\left(x^{p^{n+1}}, y^{p^{n+2}}, z_{1}^{1 / p^{\infty}}, \cdots, z_{n}^{1 / p^{\infty}}\right)$, i.e., $F \cap G=\mathbf{Z}_{p}\left(\left(x+z_{1}^{p^{2}} y^{p}+\cdots+z_{n}^{p^{n+1}} y^{p^{n}}\right)^{p}\right.$, $\left.x^{p^{n+1}}, y^{p^{n+2}}, z_{1}^{1 / p^{\infty}}, \ldots, z_{n}^{1 / p^{\infty}}\right) \subset k^{p} \Rightarrow a \in k^{p}$. Contradiction.

(d) The next counterexample shows (using the terminology of Theorem 11) that in general for a modular extension $K$ over $k, \bigcap k K^{p^{i}} \otimes M \neq K$.

Set $k=\mathbf{Z}_{p}\left(x, a_{1}^{p}, a_{2}^{p^{2}}, a_{3}^{p^{3}}, \cdots\right)$ where $x, a_{1}, a_{2}, \cdots$ are indeterminates. Set

$$
\begin{aligned}
k^{1 / p} \cap K & =k\left[x^{1 / p}, a_{1}, a_{2}^{p}, a_{3}^{p^{2}}, \ldots\right] \\
k^{1 / p^{2}} \cap K & =k\left[x^{1 / p^{2}} a_{1}^{1 / p}, a_{1}, a_{2}, a_{3}^{p}, a_{4}^{p^{2}}, \ldots\right] \\
k^{1 / p^{3}} \cap K & =k\left[x^{1 / p^{3}} a_{1}^{1 / p^{2}} a_{2}^{1 / p}, a_{1}, a_{2}, a_{3}, a_{4}^{p}, a_{5}^{p^{2}}, \ldots\right]
\end{aligned}
$$

$K$ is modular since

$$
\begin{aligned}
k^{1 / p^{n}} \cap K & =k\left[x^{1 / p^{n}} a_{1}^{1 / p^{n-1}} \ldots a_{n-1}^{1 / p}, a_{1}, a_{2}, \ldots, a_{n}, a_{n+1}^{p}, a_{n+2}^{p^{2}}, \ldots\right] \\
& =k\left[x^{1 / p^{n}} a_{1}^{1 / p^{n-1}} \ldots a_{n-1}^{1 / p}\right] \otimes k\left[a_{1}\right] \otimes k\left[a_{2}\right] \otimes \cdots \otimes k\left[a_{n}\right] \otimes k\left[a_{n+1}^{p}\right] \otimes \cdots
\end{aligned}
$$

We will show that $\bigcap k K^{p^{i}}=k$. Using the terminology of The orem 11 , we set 
$A_{i}=\left\{a_{i}\right\}$. Hence we will have $\left(\bigcap k K^{p^{i}}\right) \otimes k\left[A_{1} \cup A_{2} \cup \ldots\right]=k\left[a_{1}, a_{2}, \ldots\right] \neq K$.

Since it takes only one element to complete $\left\{a_{1}, a_{2}^{p}, a_{3}^{p^{2}}, \ldots\right\}$ to a $p$-basis for $k^{1 / p} \cap K$ over $k$, and since $\cap k K^{p^{i}}$ is modular (by Proposition 9), if

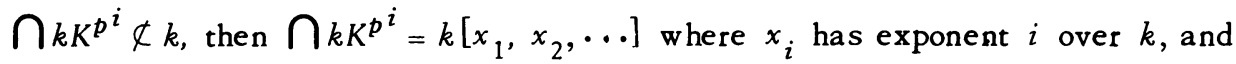
$x_{i}^{p} \in k\left[x_{1}, \cdots, x_{i-1}\right]$. Consider $K$ as lying in the perfect closure $L$ of $k . x_{1} \epsilon$ $k\left[x^{1 / p}, a_{1}, a_{2}^{p}, \cdots, a_{n}^{p^{n-1}}\right]$ for some finite $n$, so

$$
\begin{aligned}
x_{n+2} & \in\left(k^{1 / p^{n+1}} \cap L\right)\left[x^{1 / p^{n+2}}, a_{1}^{1 / p^{n+1}}, a_{2}^{1 / p^{n}}, \ldots, a_{n}^{\left.1 / p^{2}\right]}\right. \\
& =Z_{p}\left(x^{1 / p^{n+2}}, a_{1}^{1 / p^{n+1}}, a_{2}^{1 / p^{n}}, \cdots, a_{n}^{1 / p^{2}}, a_{n+1}, a_{n+2}^{p}, a_{n+3}^{p^{2}}, \ldots\right) \quad(=F) .
\end{aligned}
$$

We also have that

$$
\begin{aligned}
x_{n+2} \in k^{1 / p^{n+2}} & \cap K \\
& =\mathbf{Z}_{p}\left(x^{1 / p^{n+2}} a_{1}^{1 / p^{n+1}} a_{2}^{1 / p^{n}} \cdots a_{n}^{1 / p^{2}} a_{n+1}^{1 / p}\right. \\
& \left.a_{1}, a_{2}, \ldots, a_{n+2}, a_{n+3}^{p}, a_{n+4}^{p^{2}}, \ldots\right) \quad(=G) .
\end{aligned}
$$

Consider the following diagram of $\mathrm{Z}_{p}\left(x^{1 / p^{n+2}}, a_{1}^{1 / p^{n+1}}, a_{2}^{1 / p^{n}}, \cdots, a_{n}^{1 / p^{2}}, a_{n+1}^{1 / p}\right.$, $\left.a_{n+2}, a_{n+3}^{p}, \cdots\right)$ over $\mathrm{Z}_{p}\left(x, a_{1}, a_{2}, \ldots, a_{n+1}, a_{n+2}^{p}, a_{n+3}^{p^{2}}, \ldots\right)$.

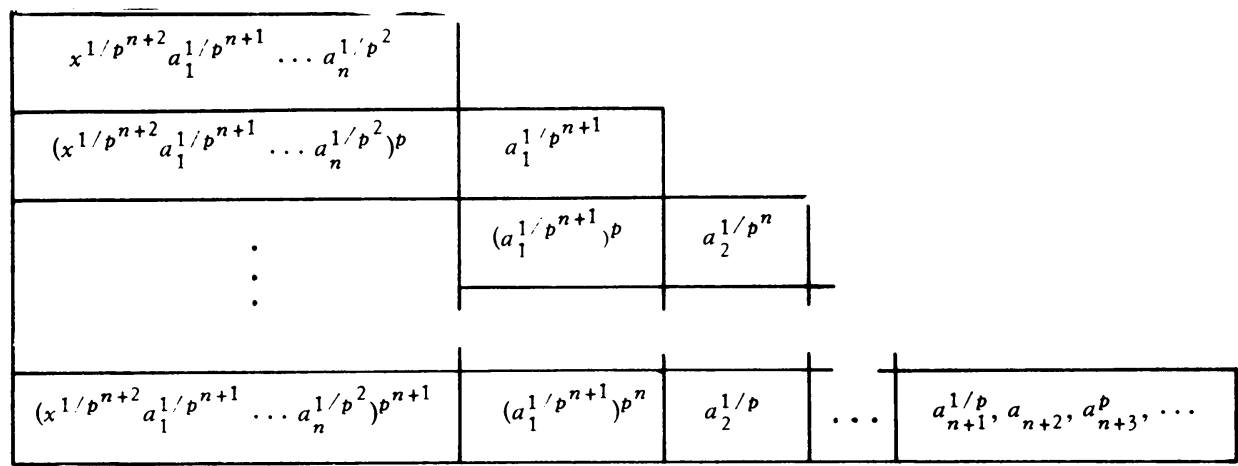

The above would be for a diagram for $F$ if we deleted the far right-hand box (containing $\left.a_{n+1}^{1 / p}, a_{n+2}, a_{n+3}^{p}, \cdots\right)$.

$G$ consists of the span of monomials in $\left(\left(x^{1 / p^{n+2}} a_{1}^{1 / p^{n+1}} \cdots a_{n}^{1 / p^{2}}\right) \cdot a_{n+1}^{1 / p}\right)^{i}$, $a_{n+2}^{i},\left(a_{n+3}^{p}\right)^{i}, \ldots$ and $\left(\left(x^{1 / p^{n+2}} a_{1}^{1 / p^{n+1}} \ldots a_{n}^{1 / p^{2}}\right)^{p}\right)^{j}$ where $i=0, \cdots, p-1$ and $j=0, \cdots, p^{n+1}-1$. So $F \cap G=\mathbf{Z}_{p}\left(\left(x^{1 / p^{n+2}} a_{1}^{1 / p^{n+1}} \cdots a_{n}^{1 / p^{2}}\right)^{p}, a_{1}, a_{2}, \cdots\right.$, $\left.a_{n+1}, a_{n+2}^{p}, a_{n+3}^{p^{2}}, \cdots\right) .[F \cap G: k]=p^{n+1} \Rightarrow\left[k\left[x_{n+2}\right]: k\right] \leq p^{n+1}$, with contradicts our initial assumption that $\left[k\left[x_{n+2}\right]: k\right]=p^{n+2}$

(e) This final counterexample shows that in general $k\left[\bigcap K^{p^{i}}\right]$ does not have a tensor complement over $k$ in $\bigcap k K^{p^{i}}$ ( $K$ over $k$ a modular extension).

We let $k=\mathbf{Z}_{p}\left(x, y, z_{1}, z_{2}, \cdots\right)$ where $x, y, z_{1}, z_{2}, \cdots$ are indeterminates. Let 


$$
\begin{aligned}
k^{1 / p} \cap K & =k\left[\left\{z_{j}^{1 / p}\right\}, x^{1 / p}\right] \\
k^{1 / p^{2}} \cap K & =k\left[\left\{z_{j}^{1 / p^{2}}\right\}, x^{1 / p^{2}}+z_{1}^{1 / p} y^{1 / p}\right] \\
k^{1 / p^{3}} \cap K & =k\left[\left\{z_{j}^{1 / p^{3}}\right\}, x^{1 / p^{3}}+z_{1}^{1 / p^{2}} y^{1 / p^{2}}+z_{2}^{1 / p} y^{1 / p}\right]
\end{aligned}
$$

Clearly $k\left[\left\{z_{j}^{1 / p^{\infty}}\right\}\right] \dot{\subset} k\left[\bigcap K^{p^{i}}\right]$. We assert that $k\left[\left\{z_{j}^{1 / p^{\infty}}\right\}\right]$ has no tensor complement in $\bigcap k K^{p^{i}}$. This will imply that $k\left[\left\{z_{j}^{1 / p^{\infty}}\right\}\right]=k\left[\bigcap K^{p^{i}}\right]$, and in particular be another counterexample showing that in general $\cap k K^{p^{i}} \neq k\left[\cap K^{p^{i}}\right]$.

Assume that there exists an $M \subset K$ such that $M \otimes_{k} k\left[\left\{z_{j}^{1 / p^{\infty}}\right\}\right] \cong K$. Since it takes only one element to complete $\left\{z_{j}^{1 / p^{n}}\right\}$ to a $p$-basis for $k^{1 / p^{n}} \cap K$ over $k^{1 / p^{n-1}} \cap K, M$ must be of the form $k\left[x_{1}, x_{2}, \ldots\right]$ where $x_{i}$ has exponent $i$ over $k$, and $x_{i}^{p} \in k\left[x_{1}, \cdots, x_{i-1}\right]$.

$x_{1} \in \mathbf{Z}_{p}\left(x^{1 / p}, y, z_{1}^{1 / p}, \cdots, z_{n}^{1 / p}\right)$ for some finite $n \Rightarrow x_{n+7} \epsilon$ $\mathbf{Z}_{p}\left(x^{1 / p^{n+2}}, y^{1 / p^{n+1}}, z_{1}^{1 / p^{n+2}}, \cdots, z_{n}^{1 / p^{n+2}}\right)(=F)$. 3ut

$$
\begin{aligned}
& x_{n+2} \in k^{1 / p^{n+2}} \cap K=Z_{p}\left(x^{1 / p^{n+2}}+z_{1}^{1 / p^{n+1}} y^{1 / p^{n+1}}+\cdots\right. \\
& \left.+z_{n+1}^{1 / p} y^{1 / p}, y, z_{1}^{1 / p^{n+2}}, z_{2}^{1 / p^{n+2}}, \ldots\right) \\
& \Rightarrow x_{n+2} \in \mathbf{Z}_{p}\left(x^{1 / p^{n+2}}+z_{1}^{1 / p^{n+1}} y^{1 / p^{n+1}}+\cdots\right. \\
& \left.+z_{n+1}^{1 / p} y^{1 / p}, y, z_{!}^{1 / p^{n+2}}, \cdots, z_{n}^{1 / p^{n+2}}, z_{n+1}^{1 / p}\right) \quad(=G) .
\end{aligned}
$$

Consider the following diagram of $\mathbf{Z}_{p}\left(x^{1 / p^{n+2}}, y^{1 / p^{n+1}}, z_{1}^{1 / p^{n+2}}, \cdots, z_{n}^{1 / p^{n+2}}, z_{n+1}^{1 / p}\right)$ over $\mathbf{Z}_{p}\left(x, y, z_{1}^{1 / p^{n+2}}, \cdots, z_{n}^{1 / p^{n+2}}, z_{n+1}\right)$.

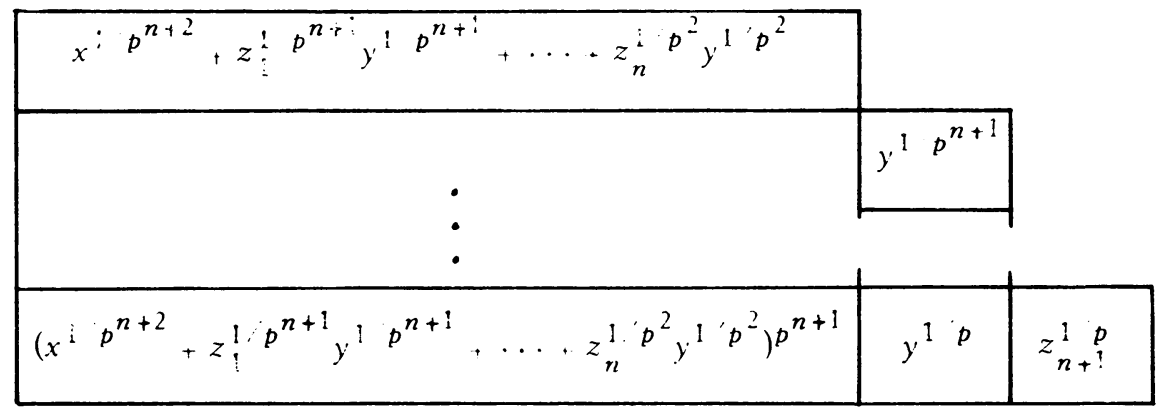

$F$ consists of the $\mathrm{Z}_{p}\left(x, y, z_{1}^{1 / p^{n+2}}, \ldots, z_{n}^{1 / p^{n+2}}\right)$ span of monomials in which (in terms of the above modular basis) no power of $z_{n+1}^{1 / p}$ occurs. $G$ consists of polynomials in $\left[\left(x^{1 / p^{n+2}}+z_{1}^{1 / p^{n+1}} y^{1 / p^{n+1}}+\cdots+z_{n}^{1 / p^{2}} y^{1 / p^{2}}\right)+z_{n+1}^{1 / p} y^{1 / p}\right]^{i}$, $\left(z_{n+1}^{1 / p}\right)^{i}(i=0, \cdots, p-1)$ and $\left(x^{1 / p^{n+2}}+z_{1}^{1 / p^{n+1}} y^{1 / p^{n+1}}+\cdots+z_{n}^{1 / p^{2}} y^{1 / p^{2}}\right)^{p \cdot j}$ $\left(j=0, \cdots, p^{n+1}-1\right)$. Hence 


$$
\begin{aligned}
& F \cap G=Z_{p}\left(\left(x^{i / p^{n+2}}-z_{1}^{1 / p^{n+1}} y^{1 / p^{n+1}}+\cdots+z_{n}^{1 / p^{2}} y^{1 / p^{2}}\right)^{p},\right. \\
& \left.y, z_{1}^{1 / p^{n+2}}, \cdots, z_{n}^{1 / p^{n+2}}\right) \text {. }
\end{aligned}
$$

Then

$$
x_{n+2}^{p^{n+1}} \in k\left[z_{1}^{1 / p}, \ldots ; z_{n}^{1 / p}\right] \Rightarrow x_{1} \in k\left[z_{1}^{1 / p}, \cdots, z_{n}^{1 / p} \mathrm{j} \Rightarrow x_{1} \in k\right.
$$

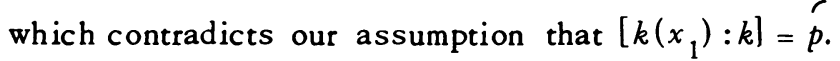

\section{BIBLIOGRAPHY}

1. 'N. Jacobson, Lectures in abstract algebra. Vol. 3: Theory of fields and Galois theory, Van Nostrand, Princeton, N. J., 1964. MR 30 \# 3087.

2. M. Sweedler, Structure of inseparable extensions, Ann. of Math. (2) 87 (1968), 401-410. MR $36 \# 6391$. 02115

DEPARTMENT OF MATHEMATICS, BOSTON STATE COLLEGE, BOSTON, MASSACHUSETTS 